Preprint typeset in JHEP style - HYPER VERSION

hep-th/0604045

UPR-T-1150

\title{
Information recovery from black holes
}

\author{
Vijay Balasubramanian \\ David Rittenhouse Laboratories, University of Pennsylvania, \\ Philadelphia, PA 19104, USA \\ vijay@physics. upenn. edu \\ Donald Marolf \\ Physics Department, UCSB, Santa Barbara, \\ CA 93106, USA \\ marolf@physics.ucsb.edu

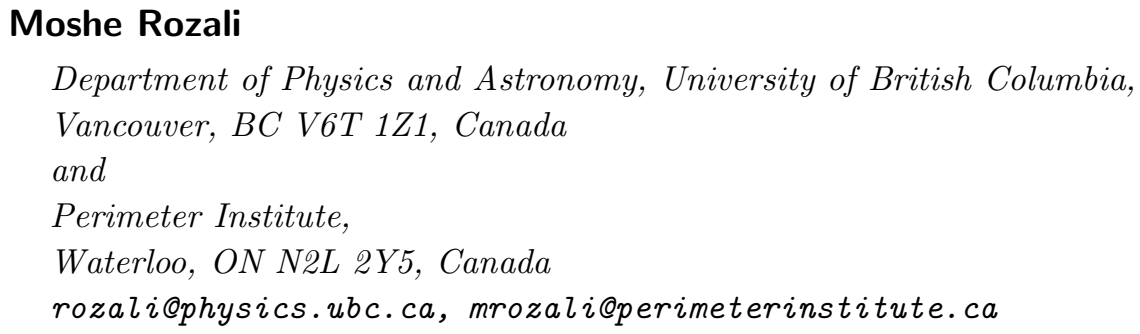

ABSTRACT: We argue that if black hole entropy arises from a finite number of underlying quantum states, then any particular such state can be identified from infinity. The finite density of states implies a discrete energy spectrum, and, in general, such spectra are non-degenerate except as determined by symmetries. Therefore, knowledge of the precise energy, and of other commuting conserved charges, determines the quantum state. In a gravitating theory, all conserved charges including the energy are given by boundary terms that can be measured at infinity. Thus, within any theory of quantum gravity, no information can be lost in black holes with a finite number of states. However, identifying the state of a black hole from infinity requires measurements with Planck scale precision. Hence observers with insufficient resolution will experience information loss.

KEYWORDS: Black holes, black hole information. 


\section{Contents}

1. Introduction 1

2. Information recovery 2

3. The absence of an unobservable interior 5

4. Discussion 5

\section{Introduction}

There are two central questions concerning the quantum physics of black holes. First, why do classical black holes apparently have a finite entropy equal to a quarter of the horizon area? Second, does information escape from an evaporating black hole, and if so, how? One might suppose that the answers to these two questions would require separate inputs from new physics. However, here we argue that any quantum mechanical theory of gravity that explains the finite entropy of black holes as the coarse grained description of a large number of microstates must also permit measurement of these states at infinity.

The basic argument is simple. If a black hole represents a finite number of states, $N \sim e^{S_{B H}}$, then the energy spectrum of the black hole must be discrete. In general such discrete spectra are quantum-mechanically non-degenerate, except as determined by symmetries of the system. Knowledge of the precise energy, along with the other commuting conserved charges, thus determines the quantum state. But since, in generic gravitating theories, charges (such as the energy) are given by boundary terms, this leads to the remarkable conclusion that complete knowledge of the black hole state is contained in the asymptotic region ${ }^{1}$.

Below we elaborate upon this observation, and argue that the relevant asymptotic measurements will always involve either very short distances that vanish as $\hbar \rightarrow 0$ or very long time scales that diverge in this limit. Either way, a conventional local, classical observer cannot measure the internal state of a black hole, although the information is present in the asymptotic region, and can be measured by observers with more sensitive instruments. We also explore why "internal observables" containing information inaccessible to the asymptotic observer do not exist, despite their apparent presence in effective field theory.

\footnotetext{
${ }^{1}$ For some (singular) extremal black holes, this conclusion is also implied by, but not dependent on, the recent observation that these spacetimes admit a classical moduli space of non-singular, horizon-free supergravity microstates which respond to most probes as if they are singular black holes [1, 2, 3, 那.
} 


\section{Information recovery}

Uncharged black holes in asymptotically flat space do not come to equilibrium with their radiation and eventually evaporate completely. Thus, in order to discuss information recovery from black holes it is helpful to begin by placing black holes "in a box" so that an equilibrium configuration of a black hole microstate accompanied by a bath of radiation can exist. The covariant method of achieving this is to consider black holes in a universe with a negative cosmological constant.

An infrared cutoff, such as the one produced by the curvature arising from a negative cosmological constant, removes the obvious continuum in the spectrum of fields associated with translational symmetry. Thus, also assuming that black holes have a finite number of microstates, the entire gravitating system has a finite number of states below any energy $E$. Due to the rapid growth of the Bekenstein-Hawking entropy, one expects that at sufficiently high energies the entropy of such systems is dominated by black holes. The typical state then involves a very heavy black hole in equilibrium with a small amount of radiation.

We will argue that complete information concerning the microstates of such black holes is available in the asymptotic region of spacetime. The central point is that, in a generic gravitating system, the energy is determined at infinity. As with the familiar ADM energy in asymptotically flat spacetimes, bulk contributions to the energy vanish due to the gravitational constraints. Thus, the energy is given entirely by a surface term.

We begin by discussing black holes in energy eigenstates. In quantum mechanics, a discrete spectrum is generically non-degenerate, except as determined by symmetries. Thus, a precise measurement of the energy (and other conserved charges) of a black hole spacetime is sufficient to identify any particular energy eigenstate.

However, such a measurement will require precision that grows exponentially in $1 / \hbar$. To see this, we temporarily ignore any additional conserved charges. Let us now suppose that a measuring device with an energy resolution $\Delta E$ interacts with a gravitating system of total energy $E$ and entropy $S(E)$. By the usual statistical mechanical understanding of entropy, this means that the number of states between $E$ and $E+\Delta E$ is $\sim e^{S}$. Since our system has a non-degenerate spectrum, the energy level spacing between $E$ and $E+\Delta E$ must be

$$
\delta E \sim \Delta E e^{-S} .
$$

In the black hole dominated regime, the density of states is given by

$$
\frac{d N}{d E} \approx \frac{d e^{S_{B H}}}{d E}=e^{S_{B H}} \frac{d S_{B H}}{d E}
$$

where $S_{B H}$, the black hole entropy, grows as some power of the energy $E$. A measuring device with an energy resolution $\Delta E$ will interact with

$$
N(E) \Delta E \approx e^{S_{B H}} \times \Delta E \frac{d S_{B H}}{d E}
$$

states. The associated entropy,

$$
\ln (N(E) \Delta E)=S_{B H}+\ln (\Delta E)+\ln \left(\frac{d S_{B H}}{d E}\right)
$$


is same as the entropy of the black hole up to logarithmic corrections. Thus the coarsegrained entropy measured by a device with resolution $\Delta E$ will equal the black hole entropy to leading order.

Choosing $\Delta E$ to be any power law in $E$ provides a coarse-graining which gives rise to the large entropy associated with the black hole. Nevertheless, measuring the energy of the state with a much greater precision $\delta E \sim \exp \left(-S_{B H}\right)$ would determine either a single state, or a small number of degenerate states which can be identified through measurements of other conserved charges. However, the Heisenberg uncertainty principle dictates that any such measurement must extend over an enormous length of time:

$$
\delta t \sim \frac{1}{\delta E} \sim \exp \left(S_{B H}\right) .
$$

In the classical limit, $\hbar \rightarrow 0$ or $\ell_{p} \rightarrow 0$, this timescale diverges exponentially because

$$
S_{B H}=\frac{A}{4 \ell_{p}^{2}}+\text { corrections } .
$$

The timescale (2.5) is comparable to the system's Heisenberg recurrence time, over which a generic state in the interval $\Delta E$ develops a matrix element of order one with any other such state $^{2}$. It is also the timescale over which large thermal fluctuations may occur, perhaps replacing the black hole by a ball of expanding hot gas. While the the gas will re-collapse to form another black hole on a (relatively) short timescale, in the meantime it is plausible that the details of the black hole's internal state are clearly visible from infinity. Thus, in retrospect it is perhaps not surprising that an experiment lasting a time $\delta t \sim \exp \left(S_{B H}\right)$ can identify the internal state of a black hole ${ }^{3}$.

We can now consider the possibility of additional conserved charges that commute with the energy. Charges associated with gauge symmetries (e.g., angular momentum, electromagnetic charges etc.) can clearly be measured at infinity in the usual ways. While any charges that are not coupled to long range gauge fields could result in degeneracies that cannot be disentangled, such degeneracies will be small because representations of typical symmetry groups do not grow exponentially quickly ${ }^{4}$. The dominance of the energy can also be seen from the fact that, in black hole thermodynamics, fixing both the mass $M$ and taking the angular momentum to vanish leads to the same entropy (to leading order) as specifying only the mass $M$ and leaving the angular momentum unconstrained. Thus the overwhelming majority of the information is available at infinity in the energy spectrum.

So far we have discussed black holes in energy eigenstates. We now turn to general superpositions

$$
|\psi\rangle=\sum_{n} a_{n}\left|E_{n}\right\rangle .
$$

\footnotetext{
${ }^{2}$ The importance of recurrence times in discussions of gravitational entropy has been highlighted in, e.g., [5, 6, 6, 8, 8, 10]. The Heisenberg time is discussed in 10].

${ }^{3}$ It has been shown that the states of certain (singular) black holes can be detected by asymptotic measurements made over such exponentially long timescales 4 .

${ }^{4}$ In fact, such charges can sometimes be measured from infinity. Examples include the asymptotic detection of black hole "hair" arising from underlying discrete symmetries (e.g. 11]), supersymmetry [4] and integrability $[12$.
} 
As is always the case for quantum systems, one cannot experimentally determine the values of all the coefficients $a_{n}$ given only a single black hole on which to perform measurements. Thus, to demonstrate whether the information is available at infinity, we should ask whether one can measure the $a_{n}$ to arbitrary accuracy given a large number of black holes prepared identically in the state $|\psi\rangle$. The frequency with which measurement of the energy gives the result $E_{n}$ yields the magnitudes $\left|a_{n}\right|^{2}$. What remains is to obtain phase information. As usual, phase information can be associated to measurement of an operator $\mathcal{B}$ which does not commute with the energy, i.e., time-dependent operators. Examples of such observables are boundary values of fields at asymptotic infinity.

For simplicity, let us choose the state $|\psi\rangle$ to be a superposition of only two energy eigenstates $|\psi\rangle=a_{1}\left|E_{1}\right\rangle+a_{2}\left|E_{2}\right\rangle$. If we now repeatedly measure the value of $\mathcal{B}$ we obtain its eigenvalues $B_{n}$ with some frequency. Consider two such eigenvalues $B_{1}$ and $B_{2}$ and the associated eigenstates

$$
\begin{aligned}
& \left|B_{1}\right\rangle=\sum_{n} b_{1, n}\left|E_{n}\right\rangle, \\
& \left|B_{2}\right\rangle=\sum_{n} b_{2, n}\left|E_{n}\right\rangle .
\end{aligned}
$$

The coefficients $b_{m, n}$ are determined by the underlying theory; we take to be known quantities. The relative frequencies of measurement of $B_{1}$ and $B_{2}$ are determined by the overlaps

$$
\begin{aligned}
& \left|\left\langle B_{1} \mid \psi\right\rangle\right|^{2}=\left|a_{1} b_{1,1}+a_{2} b_{1,2}\right|^{2} \\
& \left|\left\langle B_{2} \mid \psi\right\rangle\right|^{2}=\left|a_{1} b_{2,1}+a_{2} b_{2,2}\right|^{2} .
\end{aligned}
$$

The ratio of these two frequencies depends on both the magnitudes and the phases of $a_{1}$ and $a_{2}$. The phase dependence arises because $[\mathcal{B}, H] \neq 0$ and therefore the $b_{i, j}$ are generically non-zero. Since the $b_{i, j}$ are known, and the magnitudes $\left|a_{i}\right|$ were already determined by measurements of the energy, the relative frequency of $B_{1}$ and $B_{2}$ allows us to ascertain the relative phase of $a_{1}$ and $a_{2}$. For a more general superposition $|\psi\rangle$, repeating similar measurements fully determines the ray in Hilbert space. In this sense, full information about the microstate is available outside the black hole ${ }^{5}$.

The chief difficulty in extending the above reasoning to asymptotically flat spacetime is that the translational symmetry results in continuous spectra. We will nevertheless assume that it is possible to interpret the entropy of a black hole in asymptotically flat space in terms of a finite number of microstates, perhaps by explicitly considering a black hole in a box, or by otherwise restricting attention to the local region surrounding the black hole. In this region we can consider both the black hole and the thermal atmosphere that it generates as it evaporates. Within this framework, there should be an approximate notion of energy and we should again expect it to have a discrete, non-degenerate spectrum. Given

\footnotetext{
${ }^{5}$ The picture of information recovery offered here differs significantly from the idea explored in 5 , 13, 8, 9, 10 that a summation over multiple classical saddle points with the same asymptotic boundary conditions might allow for information recovery. Indeed, [8, 9, 10] showed in explicit examples that this mechanism was insufficient. Rather, our perspective is consistent with [3], where information is lost simply by the erasure of quantum mechanical detail in semiclassical measurements.
} 
this discreteness, measurements analogous to those described above will identify the black hole microstates.

\section{The absence of an unobservable interior}

We have argued that, in any quantum mechanical theory of gravity in which black holes have a finite number of internal states, one expects all information about the state to be available near infinity. This conclusion can be stated in terms of the commutator of operators: because the spectrum of the Hamiltonian is non-degenerate, all observables which commute with the Hamiltonian are in fact functions of the Hamiltonian itself.

There may appear to be a tension between this observation and the fact that, in classical general relativity, there are independent observables localized inside the black hole. Because the interior is causally separated from infinity, such observables commute with all asymptotic quantities. However, in our picture this is an artifact of the strict classical limit. Recall that the classical description of black holes in the $\hbar \rightarrow 0$ limit replaces very long timescales of order $e^{1 / \hbar}$ by infinity. Thus probe measurements of the sort necessary to resolve the states of a black hole are unavailable in the classical limit. As such, the usual picture of the black hole with a causally disconnected interior is the correct effective classical description. Even when $\hbar \neq 0$ this remains the effective description for semiclassical observers lacking the measurement precision necessary to resolve the microstates.

Similarly, one can readily imagine that for some class of operators $\{\mathcal{O}\}$, the commutators with Hamiltonian $H$ simply vanish rapidly in the classical limit, leading to an approximate notion of a causally separated region. However, in our picture none of these commutators vanishes exactly for $\hbar \neq 0$. How might this be explained in semiclassical terms? Consider local quantum field theory on a fixed black hole spacetime. In this context, there are observables $L$ that are localized inside the black hole. To promote these operators to observables in quantum gravity one must make them diffeomorphism invariant. Procedures to achieve this, such as integrating $L$ suitably over spacetime, generally lead to non-local operators which, when evaluated on particular spacetimes, receive contributions only from a small region [14]. In the classical limit this region will be contained inside the horizon. However, at finite $\hbar$ there is always some spread, which plausibly leads to non-vanishing commutators with operators near infinity. This may be related to rare large fluctuations of the black hole to a thermal-gas like state which are expected over the recurrence time (2.5) and which cause the horizon to be ill-defined when $\hbar \neq 0$.

\section{Discussion}

We have argued that if black hole entropy arises from a finite number of underlying quantum states, then, in any quantum mechanical theory of gravity, the information needed to identify a particular microstate is available at infinity. We used the fact that, in a generic gravitating theory, the energy is given by a surface term at infinity. While new physics is needed to explain why a given black holes is associated with a finite number of states, no 
further new physics is required to make information about black hole states available at infinity.

Although the information is available in our sense, there may be practical or even inprinciple limitations to recovery of the information by a physical apparatus. For example, in asymptotically flat space, one must also deal with the fact that black holes represent broad resonances as opposed to sharp energy eigenstates [15]. Even for stable black holes it is clear that, in order to separate black hole microstates, a measurement apparatus will itself require a large number of internal states ${ }^{6}$. In order to minimize back-reaction of such a system, one would need to either dilute it in space, or move it far away. Either way, the interactions of the apparatus with the black hole would be weakened, making the practical task of state identification more difficult.

\section{Acknowledgments}

We have enjoyed useful conversations with David Berenstein, Bartlomiej Czech, Jan de Boer, Veronika Hubeny, Vishnu Jejjala, Matt Kleban, Klaus Larjo, Rob Myers, Massimo Porrati, Mukund Rangamani, Simon Ross, Joan Simon, and Mark Srednicki. V.B. is supported in part by the DOE under grant DE-FG02-95ER40893, by the NSF under grant PHY-0331728 and by an NSF Focused Research Grant DMS0139799. D.M. was supported in part by NSF grants PHY0354978 and PHY99-07949, by funds from the University of California, and by funds from the Perimeter Institute of Theoretical Physics. M.R. is supported by a discovery grant from NSERC of Canada, and by funds from the Perimeter Institute. Research at the Perimeter Institute is supported in part by funds from NSERC of Canada and MDET of Ontario.

This paper was begun at the Perimeter Institute "Summer School on Strings, Gravity and Cosmology" and completed at the Arnold Sommerfeld Center's workshop on "Black Holes, Black Rings and Topological Strings". V.B. and D.M. are grateful to the organizers of both workshops for their hospitality. D.M. would also like to thank the Kavli Institute for Theoretical Physics for its hospitality during intermediate stages of this work.

\section{References}

[1] S. D. Mathur, "The fuzzball proposal for black holes: An elementary review," Fortsch. Phys. 53, 793 (2005) [arXiv:hep-th/0502050].

[2] H. Lin, O. Lunin and J. Maldacena, "Bubbling AdS space and 1/2 BPS geometries," JHEP 0410, 025 (2004) [arXiv:hep-th/0409174].

[3] V. Balasubramanian, V. Jejjala and J. Simon, "The library of Babel," Int. J. Mod. Phys. D 14, 2181 (2005) [arXiv:hep-th/0505123]; V. Balasubramanian, J. de Boer, V. Jejjala and J. Simon, "The library of Babel: On the origin of gravitational thermodynamics," JHEP 0512, 006 (2005) [arXiv:hep-th/0508023].

[4] V. Balasubramanian, P. Kraus and M. Shigemori, "Massless black holes and black rings as effective geometries of the D1-D5 system," Class. Quant. Grav. 22, 4803 (2005) [arXiv:hep-th/0508110].

\footnotetext{
${ }^{6}$ Issues associated with such measuring devices in gravity have been discussed in $[7,14$.
} 
[5] J. M. Maldacena, "Eternal black holes in Anti-de-Sitter," JHEP 0304, 021 (2003) [arXiv:hep-th/0106112].

[6] L. Dyson, J. Lindesay and L. Susskind, "Is there really a de Sitter/CFT duality," JHEP 0208, 045 (2002) [arXiv:hep-th/0202163]; L. Dyson, M. Kleban and L. Susskind, "Disturbing implications of a cosmological constant," JHEP 0210, 011 (2002) [arXiv:hep-th/0208013]

[7] T. Banks, W. Fischler and S. Paban, "Recurrent nightmares?: Measurement theory in de Sitter space. (((Z))," JHEP 0212, 062 (2002) [arXiv:hep-th/0210160].

[8] J. L. F. Barbon and E. Rabinovici, "Very long time scales and black hole thermal equilibrium," JHEP 0311, 047 (2003) [arXiv:hep-th/0308063];

[9] M. Kleban, M. Porrati and R. Rabadan, "Poincare recurrences and topological diversity," JHEP 0410, 030 (2004) [arXiv:hep-th/0407192].

[10] J. L. F. Barbon and E. Rabinovici, "Topology change and unitarity in quantum black hole dynamics," arXiv:hep-th/0503144

[11] M. J. Bowick, S. B. Giddings, J. A. Harvey, G. T. Horowitz and A. Strominger, "Axionic Black Holes And A Bohm-Aharonov Effect For Strings," Phys. Rev. Lett. 61, 2823 (1988); A. Strominger, "Statistical hair on black holes," Phys. Rev. Lett. 77, 3498 (1996) [arXiv:hep-th/9606016]; S. R. Coleman, J. Preskill and F. Wilczek, "Quantum hair on black holes," Nucl. Phys. B 378, 175 (1992) [arXiv:hep-th/9201059]; J. Preskill and L. M. Krauss, "Local Discrete Symmetry And Quantum Mechanical Hair," Nucl. Phys. B 341, 50 (1990).

[12] V. Balasubramanian, B. Czech, K. Larjo and J. Simon, "Integrability vs. information loss: A simple example," arXiv:hep-th/0602263.

[13] S. W. Hawking, "Information loss in black holes," Phys. Rev. D 72, 084013 (2005) [arXiv:hep-th/0507171].

[14] S. B. Giddings, D. Marolf and J. B. Hartle, "Observables in effective gravity," arXiv:hep-th/0512200.

[15] M. Srednicki, "On the observability of quantum information radiated from a black hole," arXiv:hep-th/0207090. 